\title{
Towards a Semantic Service Broker for Business Grid
}

\author{
Henar Muñoz Frutos \\ Telefónica Research \& Development \\ Valladolid, Spain \\ henar@tid.es
}

\begin{abstract}
The increasing number of infrastructure services requires the existence of mechanisms to discover and select services and resources, called service broker, based on customer requirements. This mechanism should improve the interoperability among customer-provider and be a backward compatible and light weight approach. The introduction of semantic annotations in service description (both functional and nonfunctional properties) as well as a conceptual model for business Grid can help to achieve them. Finally, the extension of the semantic Open Grid Service Architecture (S-OGSA) with the incorporation of the semantic service broker can incorporate the required capabilities to the Grid middleware.
\end{abstract}

Keywords: Service Broker, Business Grid, semantic annotations, ontology, Quality of Service.

\section{Introduction}

Current business Grid markets allow enterprises to collaborate in a dynamic environment in order to buy and sell resources (computation, storage, applications, etc ...). Moreover, these resources are virtualized as services called Grid services to reduce their complexity and be available through the Internet, boosting the number of available services. Due to the increase of the available services, some automatic mechanisms are required in order to simplify and reduce the human intervention in business.

The Web service technology provides an infrastructure for developing distributed systems and performing electronic business operations [1]. This technology implements the Service Oriented Computing (SOC) paradigm, which considers three main stakeholders: i) service producer, which offers, in our e, ii) service broker and iii) service consumer. In our case, the service producers, concretely infrastructure providers, offers resources as services, to service consumers which specify their requirements. In addition, the service broker, on behalf of the customer, discovers a set of available services for the service consumer with a certain level of the consumer satisfaction.

Thus, the service broker should locate services that fulfil a concrete user request normally by capabilities constrains. However with the increasing number 
of functional similar services in the Web [2], the discovery process is not able to find the best service to meet user requirements while ensuring the Quality of Service (QoS) 3]. Thus, a service selection process is required based on specified customer preferences or restrictions, which are considered as non-functional properties such as price, reputation and reliability, which are QoS attributes and may impact the quality of the service offered by a Web Service [3].

As part of the non-functional properties, some information related to the way of provisioning the service by the provider side can be useful. This is part of the Service-Level Agreement (SLA) templates, where besides service descriptions, it is defined information about the resources (hardware and software) to be provided, the quality of service (QoS) level to be maintained, etc [4]. Thus, a service specification offered by a provider can be materialized as a capability (functional description) provided and a set of non-functional constraints (QoS metrics, service provision terms) by using the TSLA files.

However, in this highly dynamic scenario, providers and customers may use different terminologies or even languages, to describe the conditions and requisites [5]. In this matter, it is required a mutual agreement on the service(s) functional and non-functional properties to develop a method for automatically matching the offers and the requests. In order to increase the interoperability among customers-providers, some works introduce semantic technologies in the definition of QoS metrics. Domingue et al [6] and Ren et al [3] present different conceptual models formed as ontologies so as to extend service descriptions. However, these solutions are not backward compatible, and demand providers to change their service offer descriptions. Moreover, they are not lightweight approaches, which increase the complexity to user in annotating services and the framework to implement such solutions.

In addition, considering lightweight approaches, the work introduced by Kopecky [7] defines the usage of semantic annotations for describing services based on the Web Service Modelling Ontology (WSMO). However, this approach lacks in the information provided by providers related to SLA. In addition, Oldham et al 8] propose the introduction of semantic annotations for QoS terms inside WS-Agreement 4, but extending the XML schemas, being an alternative not backward compatible.

Thus, our approach should be able to build a service broker with the following features: i) improve the interoperability among customer-providers, ii) be backward compatible and iii) be a lightweight approach.

\section{Proposed Approach}

The proposed approach tries to satisfy the main requirements commented above composed by a service broker model and an architecture which implements this model. In order to describe the non-functional properties (including the service provision) together service functional descriptions, we use TSLA from the WS-Agreement recommendation [4. Moreover, in order to increase the interoperability among customers and providers, we are going to introduce semantic 
technologies in terms of semantic annotations both in service description and QoS metrics in the TSLA. Semantic annotations allows to link SLA standards terms with concepts belonging to a conceptual model as part as the document 9]. The main drivers behind this approach are: i) the necessity for a backward compatible approach, so that, components non aware of semantic annotation can continue working with service descriptions and ii) a lightweight approach to perform any operation (selection, negotiation ...) at runtime [5].

\subsection{Service Broker Model}

In order to introduce the semantic annotations, the service broker model is defined by i) a specification for introducing semantic annotations, ii) a conceptual model for service descriptions formed as ontologies and iii) a service selection algorithm. The specification for Semantic Annotation for SLA (SA-SLA) is based on the W3C recommendation Semantic Annotation for Web Service Description Language (SA-WSDL) 9], which allows WS-Agreement terms (as metrics, KPI, service descriptions) to be pointed to semantic concepts from more expressive ontologies. Regarding the conceptual model, the approach tries to define a generic set of concepts (functional and non-functional) in a business Grid scenario, which are extensible, and can be customized to include domain-specific concepts. Finally, the service selection algorithm will involve i) the discovery of service regarding functional properties and ii) the selection of services based on customer' requirements in terms of non-functional properties. This algorithm is going to allow for working with imprecise requirements in order to provide a way of ranking and incorporate more amount of services in the compete market.

\subsection{Architecture}

Corcho et al [10] defines a Reference Architecture for the introduction of semantic technologies to Grid, called semantic Open Grid Service Architecture (S-OGSA). Concretely, S-OGSA defines a model, the capabilities and the mechanisms for the Semantic Grid. Thus, we can extend current S-OGSA architecture with the Semantic Grid Service Broker, being a new Semantically Aware Grid Service (SAGS), which should work with the rest of S-OGSA architecture to fulfill their complete functionality.

The service broker should work with different modules: i) a Grid service discovery and ii) a non-functional properties selector, besides all modules corresponding to the semantic infrastructure. In the broker design, we intent to create a discovery pattern that will be based on the well-known principle of the "separation of concerns". This principle will be applied decoupling the functional properties from the non functional properties matching.

\section{Methodology}

Methodologically, we propose a quantitative experimentation, obtaining results from different experiments. Concretely, for the experimentation, we are going to 
take the Virtual Scenario of the BREIN project [11. There, an engineering company (ANSYS) tries to outsource the computing infrastructure required for software simulations in order to reduce costs. Thus, due to the existence of several infrastructure providers (BSC, HLRS), the framework should be able to select the best provider in each moment which satisfy the customer's requests. Thus, for experimentation proposes, we are going to build a simulation testbed composed by a set of service descriptions and TSLA files, which are semantically annotated.

As the main three statement we want to demonstrate are: i)backward compatible, ii)light weigh and iii) improve interoperability, we have to design the experiments in order to show it. Thus, we will provide some annotated and non-annotated services with different used terminologies. Finally, the idea is to compare our approach with others like for instance WSMO, in order to analyze the advantages of our solution.

\section{Results}

In the implementation of the solution, we are following an agile project management approach, such as depicted by SCRUM and others originally developed for large scale IT development tasks. With this approach a set of goals considered relevant for the idea objectives is identified and implemented. Moreover, to have the full implementation some other external implementations or algorithms can be taken in order to be able to have early results. Table 1 can summarize the main goals defined and their current status.

Table 1. Goals in the semantic Grid service broker

\begin{tabular}{||l||l||c||l||}
\hline & Goals & Status & Comment \\
\hline 1 & SA-SLA specification & $90 \%$ & To be submitted to GRAAP (OGF) \\
\hline 2 & Ontologies & $35 \%$ & QoS ontology, service ontology. \\
\hline 3 & QoS Broker & $80 \%$ & Extension according to ontologies \\
\hline 4 & QoS selection algorithm & $0 \%$ & Including imprecise constrains \\
\hline 5 & Metrics Collection & $0 \%$ & Obtain metric value for providers \\
\hline 6 & $\begin{array}{l}\text { Functional properties dis- } \\
\text { covery }\end{array}$ & $0 \%$ & $\begin{array}{l}\text { Annotating WS-Agreement service de- } \\
\text { scription with SA-SLA }\end{array}$ \\
\hline \hline
\end{tabular}

\section{Conclusions and Future Work}

This document has established the main pillars towards the realization of a semantic grid service broker for business Grid. It has started from the definition of the context, where mechanisms are required for the discovery and selection of Grid services considering non-functional properties. Moreover, the first analysis of the state of the art pointed out that the existing approaches are not usually focused on service Grid and do not solve the heterogeneity problem planned in a open market place. Finally, other approaches, which can satisfy these requirements, lack in a backwards compatible and light weight approach, with allows 
continuing working with current components. Our proposed approach relies on the introduction of semantic annotations in TSLA and ontologies to work towards these requirements. Currently, there are some developed results as the QoS broker, but we should continue working on the state of the art to guarantee that the idea is original, as well as some bullet points marked in the proposed approach.

\section{References}

1. Papazoglou, M.P., Heuvel, W.J.: Service oriented architectures: approaches, technologies and research issues. The VLDB Journal 16(3), 389-415 (2007)

2. D'Mello, D.A., Ananthanarayana, V.S., Thilagam, S.: A qos broker based architecture for dynamic web service selection. In: AMS 2008: Proceedings of the 2008 Second Asia International Conference on Modelling \& Simulation (AMS), pp. 101106. IEEE Computer Society, Washington (2008)

3. Ren, K., Chen, J., Chen, T., Song, J., Xiao, N.: Grid-based semantic web service discovery model with qos constraints. In: SKG 2007: Proceedings of the Third International Conference on Semantics, Knowledge and Grid, Washington, DC, USA, pp. 479-482. IEEE Computer Society, Los Alamitos (2007)

4. Grid Resource Allocation Agreement Protocol WG (GRAAP-WG): Web Services Agreement Specification (WS-Agreement), Version 2005/09 (2005)

5. Kotsiopoulos, I., Juber, I.S., Tenscbert, A., Cirujeda, J.B., Koller, B.: Using semantic technologies to improve negotiation of service level agreements (2008)

6. Domingue, J., Cabral, L., Galizia, S., Tanasescu, V., Gugliotta, A., Norton, B., Pedrinaci, C.: Irs-iii: A broker-based approach to semantic web services. Web Semant. 6(2), 109-132 (2008)

7. Kopecký, J., Vitvar, T.: Wsmo-lite: Lowering the semantic web services barrier with modular and light-weight annotations. In: ICSC 2008: Proceedings of the 2008 IEEE International Conference on Semantic Computing, Washington, DC, USA, pp. 238-244. IEEE Computer Society, Los Alamitos (2008)

8. Oldham, N., Verma, K., Sheth, A., Hakimpour, F.: Semantic ws-agreement partner selection. In: WWW 2006: Proceedings of the 15th international conference on World Wide Web, pp. 697-706. ACM, New York (2006)

9. Kopecký, J., Vitvar, T., Bournez, C., Farrell, J.: Sawsdl: Semantic annotations for wsdl and XML schema. IEEE Internet Computing 11(6), 60-67 (2007)

10. Corcho, O., Alper, P., Kotsiopoulos, I., Missier, P., Bechhofer, S., Goble, C.: An overview of s-ogsa: A reference semantic grid architecture. Web Semantics: Science, Services and Agents on the World Wide Web 4(2), 102-115 (2006)

11. The BREIN Consortium: The brein project (2008) 